\title{
Tiny Tesla Turbine Analytical Performance Validation Via Dynamic Dynamometry
}

\author{
Matthew J. Traum ${ }^{1,{ }^{*}}$ and Hope L. Weiss ${ }^{2}$ \\ ${ }^{1}$ Engineer Inc., $4832 \mathrm{NW} 76^{\text {th }}$ Rd, Gainesville, FL, USA 32653 \\ ${ }^{2}$ California State University Fullerton, Mechanical Engineering Department, $800 \mathrm{~N}$ State College \\ Blvd. Fullerton, CA, USA 92831
}

\begin{abstract}
Tesla turbines produce power at high rotation rate and low torque relative to other prime movers. At a tiny scale, this attribute renders Tesla turbines poorly matched to dynamometers designed to characterize electricand gasoline-powered radio-controlled vehicles and kit cars. Techniques are needed to enable Tesla turbine design and performance evaluation. An analytical modelling approach was recently developed by Carey, and a complimentary experimental technique, dynamic dynamometry, can determine Tesla turbine power curves without a dynamometer. This paper mutually validates these approaches by comparing them to each other using results from a 3D printed 4-disk tiny Tesla turbine with characteristic disk inner/outer diameter of $11.54 \pm 0.01 \mathrm{~mm}$ and $24.85 \pm 0.01 \mathrm{~mm}$ respectively. The Carey model predicts maximum power output of $0.077 \pm 0.015 \mathrm{~W}$, and dynamic dynamometry predicts $0.122 \pm 0.008 \mathrm{~W}$, a $36.9 \%$ difference. Bounding assumptions were used and more accurate parameter measurements will drive these values closer together. Peculiarities of tiny Tesla turbine operation are also described, including the discovery that turbine spin-down rotational velocity is not linear with time. This phenomenon is likely caused by fluid boundary layer shear between the housing and outer disks. It is not observed in larger Tesla turbines, suggesting a speed, size and/or disk count threshold at which this phenomenon introduces non-trivial parasitic reduction in performance.
\end{abstract}

\section{Introduction}

Before contemporary resurgence of academic attention to Tesla turbines, this configuration captured the interest of hobbyists drawn to its ease of fabrication; in particular members of the Tesla Engine Builders Association (TEBA) [1]. Moreover, Tesla turbines can process gas, vapor, liquid, or even mixed-phase working fluids without damage. So, they can safely run on a wide variety of energy sources available in a home shop: compressed air, pressurized water, or a home-built boiler (without superheat). Combustion is not needed. Despite these benefits, Tesla turbines produce power at high rotation rate and low torque relative to other prime movers. This attribute renders them poorly matched to hobby-scale dynamometers in

* Corresponding author: $\underline{\mathrm{CEO} @ \text { EngineerInc.net }}$ 
their power range available to characterize electric- and gasoline-powered radio-controlled vehicles and kit cars.

Until recently, no reliable analytical technique to predict Tesla turbine performance from a set of preliminary design parameters existed. With no way to reliably design Tesla turbines to a target output power and with no way to experimentally characterize built Tesla turbine performance, their design and manufacture was haphazard. Builders produced Tesla turbines only to discover they did not generate the power and/or torque needed for their target application [2].

Derivation of Tesla turbine analytical modelling equations by Carey in 2009 and 2010 enabled power output prediction based on geometric parameters for the first time $[3,4]$. Certainly, Carey's initial Tesla turbine models have limitations including absence of nozzlerotor interaction and lack on insight on the influences of nozzle number, placement, or design on performance. These limitations were addressed later by Carey's research group [5-8]. However, these later improvements stripped away the closed-formed analytical solution utility of Carey's initial models, which remain useful for simple engineering design sizing and scoping of Tesla turbines. Introduction of dynamic dynamometry by Yang et al. in 2013 enabled experimental Tesla turbine performance testing without need for a specialized dynamometer $[9,10]$. These techniques give Tesla turbine builders the capability to systematically design and test their machines. This paper chronicles the first effort to compare these Tesla turbine modelling and evaluation techniques to each other for mutual validation.

\section{Background}

A rich foundation in Tesla turbines is available in a pair of review articles [11,12]. Tesla turbines rely on boundary layer flow between closely-spaced disks, which favours the low Reynolds number regime for increased energy conversion efficiency. While it is possible to lower the Reynolds number by increasing working fluid viscosity [13], working fluid properties often cannot be manipulated in real applications. So, the practical way to lower Reynolds number and increase Tesla turbine efficiency is to make the turbine as tiny as possible [14]. In response, an effort is made in this paper to work with the tiniest commercially-available Tesla turbine the authors could obtain. For a brief time, the turbine shown in Figure 1, which is 3D-printed in polylactic acid (PLA), was commercially available [15].
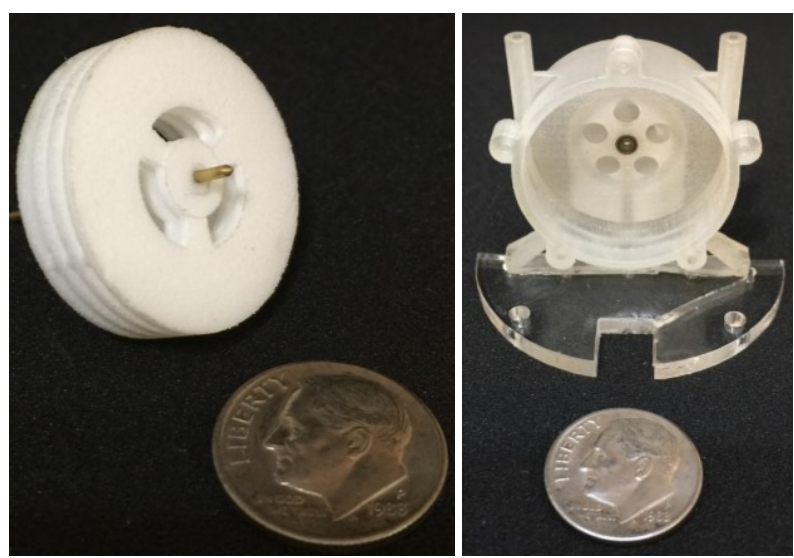

Fig. 1. 3D Printed tiny Tesla turbine made from PLA. 


\subsection{Theory}

\subsubsection{Analytical Performance Model}

Carey's technique for Tesla turbine performance modelling based on geometric parameters was a revolutionary advancement in Tesla turbine research [3,4]. Starting from the NavierStokes equations, Carey derived a closed-form analytical solution for $\widehat{W}\left(\xi, R e_{m}^{*}\right)$, the dimensionless tangential velocity difference between a Tesla turbine disk and the circulating fluid at any radial location,

$$
\widehat{W}\left(\xi, R e_{m}^{*}\right)=\frac{e^{\frac{24 \xi^{2}}{R e_{m}^{*}}}}{\xi}\left[\frac{R e_{m}^{*}}{24} e^{\frac{-24 \xi^{2}}{R e_{m}^{*}}}+\widehat{W}_{o}-\frac{R e_{m}^{*}}{24} e^{\frac{-24}{R e_{m}^{*}}}\right]
$$

where $\xi=r / r_{o}$ is the dimensionless radial disk location, $\hat{W}_{o}$ is the dimensionless relative disk/fluid velocity at $\xi=1$, and $R e_{m}^{*}$ is a modified Reynolds number:

$$
R e_{m}^{*}=\frac{2 b \dot{m}_{c}}{\pi \mu r_{o}^{2}}
$$

Here, $b$ is the inter-disk gap, $\dot{\mathrm{m}}_{c}$ is the working fluid mass flow rate per channel between disks, and $r_{0}$ is the disk outer radius. By applying Euler's Turbine Equation to Carey's model, Traum et al developed a closed-form analytical solution to predict Tesla turbine power output given only easily-measured geometric parameters [14].

\subsubsection{Dynamic Dynamometry}

Dynamic dynamometry uses the rotational inertia of the turbine spindle assembly and the friction in the bearings as the load. An optical tachometer tracks instantaneous rotational velocity, and no separate dynamometer is needed to extract power curves. The unloaded turbine is spun up at some regulator-fixed inlet gas pressure until steady state is reached. The flow is then instantly shut off, and the turbine spins back down to rest. In a separate experiment, a weight tied to a light string wrapped around the turbine spindle is dropped to interrogate spindle rotational inertia. For this paper, data were captured by videoing the optical tachometer with a stopwatch and playing back events frame-by-frame. Turbine rotation rate as a function of time, $\dot{\Theta}_{a}(t)$, was extracted. Experimental parameters were then combined with data from the initial pressurized spin-up to determine turbine power, $P_{\text {out }}$, using

$$
P_{\text {out }}=\frac{I}{\tau} \cdot\left[\dot{\Theta}_{\max } \cdot \dot{\Theta}_{a}(t)-\dot{\Theta}_{a}(t)^{2}\right]
$$

where $I$ is the turbine spindle rotational inertia, $\tau$ is the spin-up time constant, and $\dot{\Theta}_{a}(t) \&$ $\dot{\Theta}_{\text {max }}$ are the instantaneous and maximum (steady-state) turbine rotation rates, respectively. 


\subsection{Method}

Figure 2A shows the original dynamic dynamometry experimental setup published by Yang et al using a desktop-sized Tesla turbine [13,14]. This configuration inspired the dynamic dynamometry setup used for this paper, Figure 2B, which uses a 3D printed tiny Tesla turbine. The turbine's geometric parameters, including disk thickness, are summarized in Table 1. At $1.1 \pm 0.1 \mathrm{~mm}$ wide, the turbine disks are relatively thick. This feature is necessary because the disks are 3D printed and must be released from the print stage without warping.

\subsubsection{Spindle Inertia Determination}

The first dynamic dynamometry step is obtaining the spindle's rotational inertia, $I$. A strength of this technique is that $I$ can be determined in situ; the spindle need not be removed from the housing, and its geometric features need not be measured. For example, in the original dynamic dynamometry experiments of Yang et al, the Tesla turbine was never opened, and its internal disk geometry was never known. Since the tiny turbine of the current experiment can be opened, $I$ can be estimated based on measured geometry. For a hollow cylinder of non-trivial wall width and height, $h$, rotating about its central axis

$$
I=\frac{\pi \rho h}{2}\left(r_{o}^{4}-r_{i}^{4}\right)
$$

where $\rho$ is PLA density, and $r_{i} / r_{o}$ are the inner / outer disk radii. From measured disk parameters of Table $1, I \approx 4.80 \times 10^{-8} \mathrm{~kg}-\mathrm{m}^{2}$. This approximation represents a lower bound on $I$ because it does not account for rotational turbine assembly inertias of the internal spokes, the central spindle, or the external spool used to trip the optical tachometer.

Using dynamic dynamometry, turbine spin-up acceleration $\left(\ddot{\phi}_{a}\right)$ spin-down acceleration $\left(\ddot{\Theta}_{d}\right)$ and disk outer radius $\left(r_{o}\right)$ combine to yield

$$
I=\frac{\left[m g-m \ddot{\phi}_{a} \frac{r_{o}}{2}\right]\left(\frac{r_{o}}{2}\right)}{\ddot{\phi}_{a}-\ddot{\Theta}_{d}}
$$

where $m$ is the mass of a small trial weight attached to the turbine's spindle by a thin string that spins up the turbine when dropped, and $g$ is local gravitational acceleration.
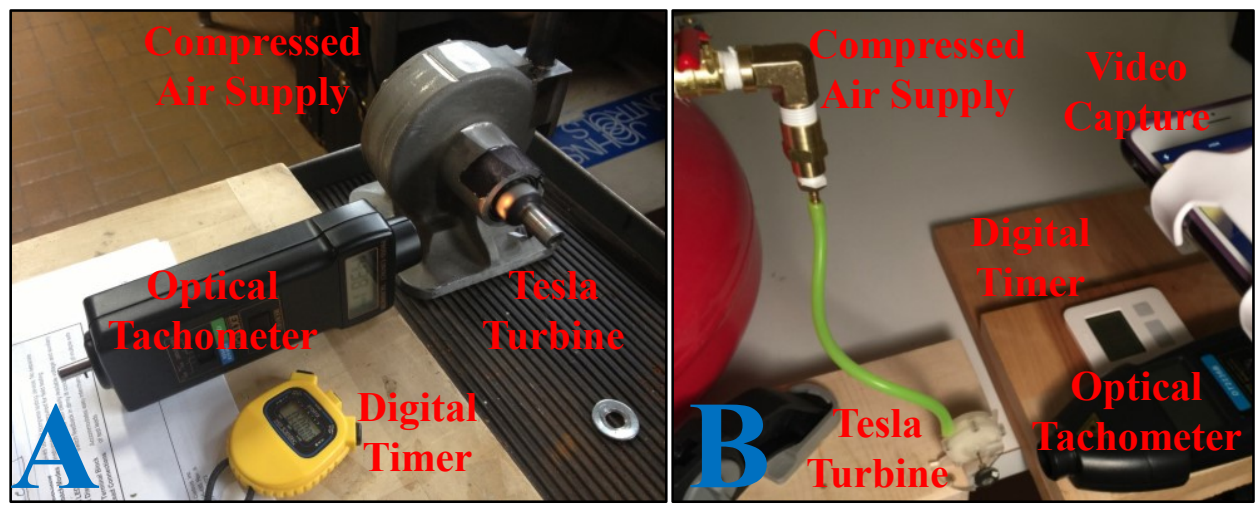

Fig. 2. (A) The original dynamic dynamometry experiment with desktop Tesla turbine $[9,10]$. (B) The dynamic dynamometry experiment analysed in this paper with a 3D printed PLA tiny Tesla turbine. 
Table 1. 3D printed tiny Tesla turbine parameters.

\begin{tabular}{|c|c|}
\hline Parameter & Parameter Values \\
\hline Disk Outer Radius, $r_{o}$ & $24.85 \pm 0.01 \mathrm{~mm}$ \\
\hline Disk Inner Radius, $r_{i}$ & $11.54 \pm 0.01 \mathrm{~mm}$ \\
\hline Disk Thickness, $h$ & $1.1 \pm 0.01 \mathrm{~mm}$ \\
\hline Inter-Disk Spacing, $b$ & $0.9 \pm 0.01 \mathrm{~mm}$ \\
\hline Number of Disks, $n$ & 4 \\
\hline PLA Density, $\rho[16]$ & $1.26 \mathrm{~kg} / \mathrm{m}^{3}$ \\
\hline
\end{tabular}

\subsubsection{Power Curve Data Collection}

To extract experimental turbine power via dynamic dynamometry, flow of compressed air through the turbine is set by fixing upstream regulator pressure to 20 PSIG $(\sim 239 \mathrm{kPa}$, absolute) with the turbine inlet valve closed. The valve to the turbine is then opened quickly, air flows through the turbine, and it spins up to steady state. Rotation rate as a function of time is monitored via optical tachometer (Fig. 2B). To determine instaneous output tower, data are input into

$$
P_{\text {out }} \approx I \cdot \frac{\Delta \dot{\Theta}_{a}(t)}{\Delta t} \cdot \dot{\Theta}_{a}(t)
$$

where $I$ is rotiational inertia, $\dot{\Theta}_{a}(t)$ is rotational acceleration, and $\Delta t$ is a time step. Experimetnal data from Eqn. 6 are validated against curves produced by Eqn. 3 for the same fixed input working fluid pressure. Given the small size of the turbine, air leakage likely occurred from the housing and through the rotating bearings. However, the magnitude and impacts of these ventilation losses were not quantified or accounted for in these experiments.

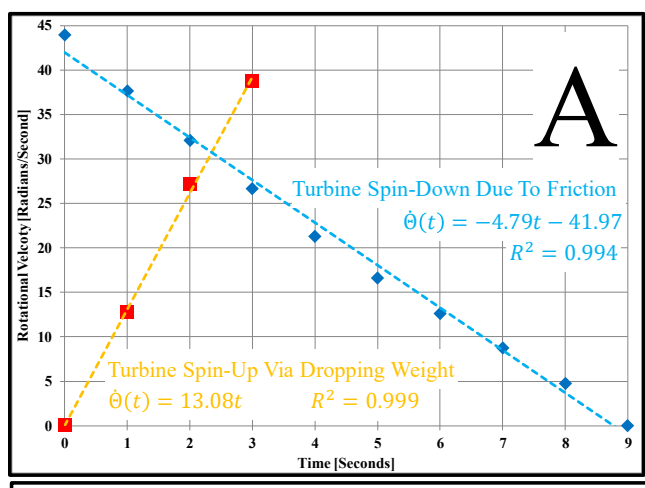

Experimental Spin-Down

\section{Experimental Spin-Up}

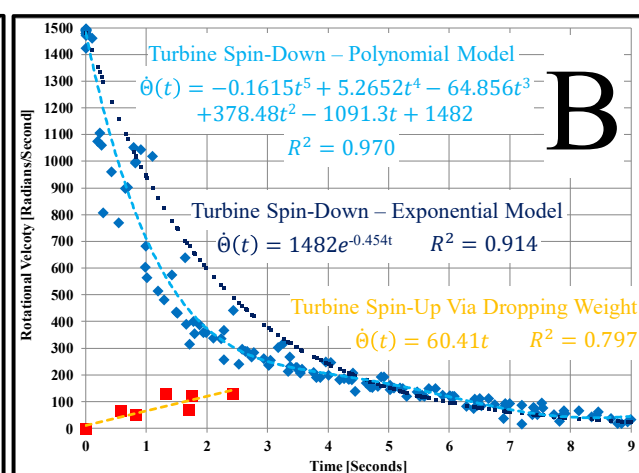

Linear Spin-Down Model Polynomial Spin-Down Model Exponential Spin-Down Model

Fig. 3. (A) Spin-Up and spin-down data from the original dynamic dynamometry experiments of Yang et al indicate these dynamic processes are linear for a benchtop-scale Tesla turbine [9]. (B) For the 3D printed tiny Tesla turbine, spin-down is non-linear at higher rotation rates.

\section{Results}

Figure 3A shows spin-up and spin-down data from the original dynamic dynamometry research of Yang et al [9]. These processes are linear, producing a constant $I$ value from Eq. 
5. Similar linear trends were expected in new experiments with the $3 \mathrm{D}$ printed tiny turbine, but the spin-down process was not linear (Fig. 3B).

Exponential-like decay in the micro-turbine spin-down data suggest a retarding torque effect beyond bearing friction assumed in the original dynamic dynamometry approach. Due to this unexpected result, $I$ rotational inertia could not be directly calculated. So, $I \approx 4.80 \mathrm{x}$ $10^{-8} \mathrm{~kg}-\mathrm{m}^{2}$, determined by geometric disk measurements (Eqn. 4), was used instead of Eqn. 5. The Standard Error of the Estimate minimizing spin-up experimental time constant is 0.22 $\pm 0.01 \mathrm{~s}$, and the Eqn. 3 maxima occurs at $748.6 \pm 0.1 \mathrm{rad} / \mathrm{s}$ rotation rate. Thus, the maximum turbine power output predicted by dynamic dynamometry for 20 PSIG input pressure is 122 $\pm 8 \mathrm{~mW}$.

The Carey model of Eqn. 1 yields a maximum theoretical power of $77 \pm 15 \mathrm{~mW}$ for this Tesla turbine geometry.

\section{Discussion}

\subsection{Microturbine Workability for Experimental Research}

Operating in the low Reynolds number regime (where boundary layer flow dominates) induces higher energy conversion efficiency favouring smaller Tesla turbines over larger ones [13]. At small scale, data sampling is a challenge due to the speed of transient processes. The update rates of the optical tachometers shown in Figure 2 are $\sim 0.25 \mathrm{~s}$. Charactering the desktop-scale turbine (Fig. 2A) at this sampling rate presented no problems, but it was too slow for direct data collection using the tiny turbine (Fig. 2B). To overcome this problem, identical spin-up/spin-down sequences were repeated multiple times, and data sets layered atop each other to provide continuity for calculation. A faster optical tachometer would eliminate need for this time-consuming data gathering approach.

\subsection{Spin-Down Curve Non-Linearity}

In the original dynamic dynamometry research of Yang et al, both the spin-up and spin-down plots were linear (Fig. 3A) over the turbine's full rotational velocity range. The tiny Tesla turbine used here demonstrated linearity during weighted spin-up, but the spin-down process was not linear. As shown in Fig. 3B, it more closely followed a trend of exponential decay. We posit two possible reasons for this difference. First, bearing friction might increase as turbine rotational velocity increases, producing more resistance at higher revolution rates. Second, shear in the boundary layer between the disks and the non-moving turbine housing induces an additional retarding force beyond bearing friction. This shear is large at high turbine rotation rates causing rapid turbine rotational deceleration, but it shrinks as the turbine slows down. Qualitatively this trend is precisely what is observed in Fig. 3B.

This phenomenon was not observed in the larger desktop-scale turbine of Yang et al. Since the only previous published dynamic dynamometry experience is the work of Yang et al, it was initially assumed that non-linear Tesla turbine spin-down was unusual. However, the nonlinear tiny Tesla turbine spin-down of Fig. 3B may be more typical than the linear spin-down in Figure. 3A for the larger Tesla turbine. Moreover, the larger turbine was spun down from a much lower $45 \mathrm{rad} / \mathrm{s}$ initial maximum rotation rate than the tiny turbine, which spun down from $1500 \mathrm{rad} / \mathrm{s}$. Dissimilar behaviours arising from disparate Tesla turbine speed, disk number, and disk diameter suggest existence of a turbine metric beyond which spin retarding effects becomes non-trivial. Indeed, a similar abrupt performance transition in rotor speed was previously observed, which might have the same underlying cause $[2,17]$. Additional study to better understand this phenomenon is required. 
Ultimately, the $I$ of Eqn. 6 could not be calculated via dynamic dynamometry because spin-down rotational acceleration was not constant. Calculated $I$ based on geometry was used instead, but it did not account for smaller turbine spindle features. The actual $I$ value will be higher than that calculated, which drives turbine power output results from dynamic dynamometry toward the Carey analytical model result.

\subsection{Empirical / Model Agreement}

Maximum turbine output power via dynamic dynamometry was $0.122 \pm 0.008 \mathrm{~W}$, and the Carey model predicted $0.077 \pm 0.015 \mathrm{~W}$, a difference of $36.9 \%$. Even considering experimental uncertainty, these values do not precisely agree. Nonetheless, given simplicity of the experimental techniques and the generalizing assumptions applied, empirical $/ \mathrm{model}$ agreement obtained through this study is remarkable. As noted above, the Carey model includes analytical simplifications that could also explain model/experiment discrepancy. The model ignores nozzle configuration as well as flow leakage out of the turbine housing and through the bearings. Nonetheless, relatively close agreement between dynamic dynamometry and the Carey model, motivates additional research to further explore mutual validation of these approaches.

\section{Conclusion}

Experimental work with a 3D-printed tiny Tesla turbine evaluated and compared maximum power output obtained via two techniques: Carey's analytical method and dynamic dynamometry. For a 4-disk Tesla turbine with characteristic outer diameter of $24.85 \pm 0.01$ $\mathrm{mm}$, the Carey model predicts maximum power output of $0.077 \pm 0.015 \mathrm{~W}$, and dynamic dynamometry predicts $0.122 \pm 0.008 \mathrm{~W}$, a $36.9 \%$ difference. Assumptions applied in the dynamic dynamometry analysis give an upper bound on power. With more sophisticated analysis, the calculated dynamic dynamometry turbine output power will approach Carey's model.

Peculiarities of experimental Tesla turbine work at small scale were also described including the discovery that tiny turbine spin-down velocity is not linear with time. This phenomenon, likely caused by boundary layer shear between the housing and outer disks, was not observed in a larger Tesla turbine spinning down from a lower starting rotational velocity. This finding suggests existence of a turbine speed, size, and/or disk count threshold beyond which this phenomenon becomes non-trivial for Tesla turbine performance.

\subsection{Future Work}

The next step in this research endeavour is to match the tiny Tesla turbine with a small generator for power generation. This arrangement will facilitate collection of experimental power curves for a range of turbine inlet pressures. Power curves obtained directly will facilitate validation of both the Carey model and dynamic dynamometry as tools for firstorder Tesla turbine design and performance analysis.

We gratefully acknowledge the Wisconsin Space Grant Consortium 2012-2013 Higher Education Incentives Award Program for financial support. We also thank Tamir Emran for his manuscript review and insightful inputs. 


\section{References}

1. J. Hayes, "Tesla Engine Builders Association Web Site," URL: www.teslaengine.org (accessed July 1, 2019)

2. T. A. Emran, R. C. Alexander, C. T. Stallings, M. A. DeMay, M. J. Traum, ASME Early Career Technical Journal, 10, pp. 158-164 (2010)

3. V. P. Carey, Proc. ASME 2009 International Mechanical Engineering Congress \& Exposition, IMECE2009-10814 (2009)

4. V. P. Carey, J. of Engineering for Gas Turbines \& Power, 132, 12, pp. 122301-1 122301-8 (2010)

5. V. P. Carey, Proc. ASME 2010 International Mechanical Engineering Congress \& Exposition, pp. 31-38 (2010)

6. V. G. Krishnan, Z. Iqbal, M. M. Maharbiz, Proc. $16^{\text {th }}$ International Solid-State Sensors, Actuators, and Microsystems Conf., pp. 1851-1854 (2011)

7. V. D. Romanin, V. G. Krishnan, V. P. Carey M. M. Maharbiz, Proc. ASME 2012 International Mechanical Engineering Congress \& Exposition, IMECE2012-89675 (2012)

8. V. G. Krishnan, V. D. Romanin, V. P. Carey, M. M. Maharbiz, J. of Micromechanics and Microengineering, 23, 12 (2013)

9. Z. Yang, H. L. Weiss, M. J. Traum, Proc. ASEE 2013 North Midwest Section Conf. (2013)

10. Z. Yang, H. L. Weiss, M. J. Traum, Proc. 23rd Annual Wisconsin Space Conf. (2013)

11. W. Rice, Proc. 4th International Tesla Symposium (1991)

12. H. E. Gupta, S. P. Kodali, Int. J. Adv. Transport Phenomena, 2, 1, pp. 7-14 (2013)

13. M. S. Alrabie, F. N. Altamimi, M. H. Altarrgemy, F. Hadi, M. K. Akbar, M. J. Traum, Proc. ASME 2017 Power \& Energy Conf., PowerEnergy2017-3442 (2017)

14. M. J. Traum, F. Hadi, M. K. Akbar, J. Energy Resources Technology, 140, 3. (2018)

15. EpicPhysics.com, "Tesla Turbine Kit", URL: www.epicphysics.com/model-enginekits/tesla-turbine-kit/ (accessed July 1, 2019)

16. Polymer Properties Database, "Polylactic acid," Chemical Retrieval on the Web (CROW), URL: https://polymerdatabase.com/polymers/polylacticacid.html (accessed July 1, 2019)

17. T. A. Emran, Master's Thesis, University of North Texas (2011) 